\title{
HYPER-WIENER INDEX OF ZIGZAG POLYHEX NANOTUBES
}

\author{
MEHDI ELIASI $^{1}$ and BIJN TAERI ${ }^{\otimes 2}$
}

(Received 18 April, 2007; revised 20 April, 2008)

\begin{abstract}
The hyper-Wiener index of a connected graph $G$ is defined as $W W(G)=$ $(1 / 4) \sum_{(u, v) \in V(G) \times V(G)}\left(d(u, v)+d(u, v)^{2}\right)$, where $V(G)$ is the set of all vertices of $G$ and $d(u, v)$ is the distance between the vertices $u, v \in V(G)$. In this paper we find an exact expression for the hyper-Wiener index of $T U H_{6}[2 p, q]$, the zigzag polyhex nanotube.
\end{abstract}

2000 Mathematics subject classification: primary 05C12; secondary 05A15.

Keywords and phrases: topological index, distance, hyper-Wiener index, nanotubes.

\section{Introduction}

Topological indices are one of the descriptors of molecules that play an important role in structure property and structure activity studies, particularly when multivariate regression analysis, artificial neural networks, and pattern recognition are used as statistical tools. The Wiener index was the first topological index that was introduced in 1947 by Harold Wiener. He published a series of papers [27-31] showing that there are excellent correlations between the Wiener index and a variety of physicochemical properties of organic compounds. For a nice survey on this topic we encourage the reader to consult $[13,14]$.

Let $G$ be an undirected connected graph without loops or multiple edges. The set of vertices and edges of $G$ are denoted by $V(G)$ and $E(G)$, respectively. For vertices $x$ and $y$ in $V(G)$, we denote by $d(x, y)$ (or $d_{G}(x, y)$ when we deal with more than one graph) the topological distance, that is, the number of edges on a shortest path, joining

\footnotetext{
${ }^{1}$ Department of Mathematical Sciences, Isfahan University of Technology, Isfahan, 84156-83111, Iran; e-mail: eliasi@math.iut.ac.ir.

${ }^{2}$ Department of Mathematical Sciences, Isfahan University of Technology, Isfahan, 84156-83111, Iran; e-mail: b.taeri@cc.iut.ac.ir.

(C) Australian Mathematical Society 2009, Serial-fee code 1446-1811/09 \$16.00
} 


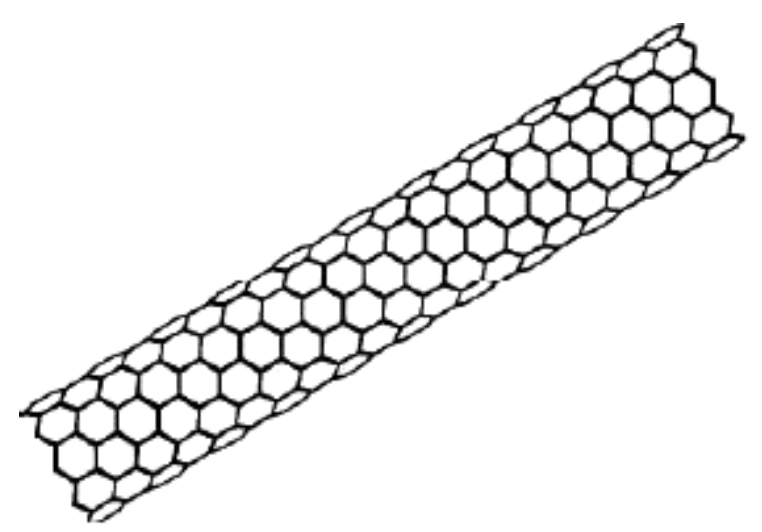

FIGURE 1. A $T U \mathrm{HC}_{6}[2 p, q]$ nanotube.

two vertices of $G$. The Wiener index of a graph $G$ is the half sum of all distances in the graph $G$ :

$$
W(G)=\frac{1}{2} \sum_{(u, v) \in V(G) \times V(G)} d(u, v) .
$$

The hyper-Wiener index is one of the recently conceived distance-based graph invariants, used as a structure-descriptor for predicting physicochemical properties of organic compounds (often significant for pharmacology, agriculture and environmental protection). Randić in [26] introduced an extension of the Wiener index for trees, and this has come to be known as the hyper-Wiener index. Klein et al. [25] generalized this extension to cyclic structures as

$$
W W(G)=\frac{1}{2} W(G)+\frac{1}{4} \sum_{(u, v) \in V(G) \times V(G)} d(u, v)^{2} .
$$

The hyper-Wiener index $W W(G)$ has seen widespread use in correlations; references may be found in [4] and also in [24]. In [5, 6], Diudea has treated both $W(G)$ and $W W(G)$ in a common matrix framework.

In a series of papers, Diudea and coauthors [7-12, 23] computed the Wiener index of some nanotubes as did the present authors in [15-22].

In this paper we find an exact expression for the hyper-Wiener index of the zigzag polyhex nanotubes of circumference $2 p$ and length $q$, denoted by $G:=$ $T U \mathrm{HC}_{6}[2 p, q]$. (An example is shown in Figure 1.) For this purpose we choose coordinate labels for vertices of $G$ as shown in Figure 2. In [15] we have included a MATHEMATICA [32] program to produce the graph of $T U H C_{6}[2 p, q]$. With this program we can compute the hyper-Wiener indices of the graphs under consideration.

We note that $G$ is a bipartite graph which means that its vertices can be coloured with white and black so that adjacent vertices have different color, or equivalently, that 


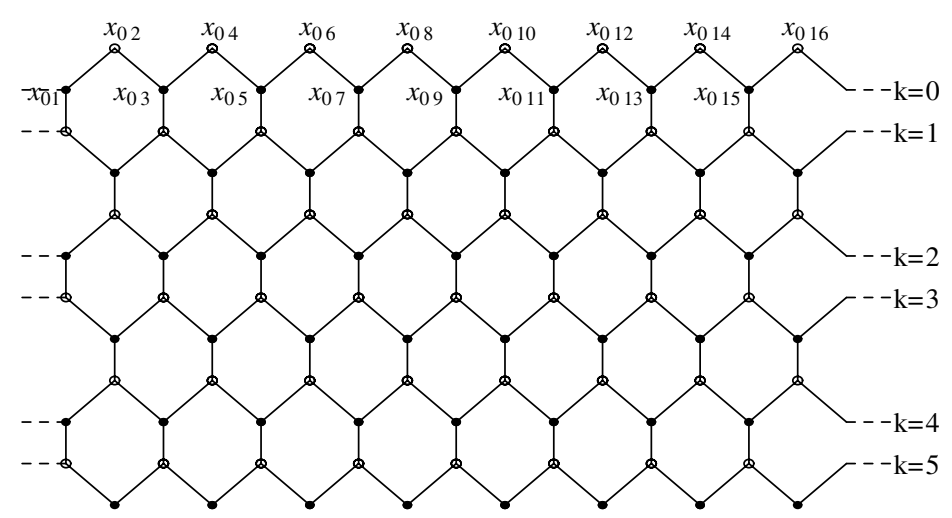

FIGURE 2. A $T U H_{6}[2 p, q]$ lattice with $p=8$ and $q=6$.

every cycle has even length (see [18, Theorem 2.4]). Obviously the number of vertices and the number of edges of $G$ is $n=|V(G)|=2 p q$ and $m=|E(G)|=3 p q-p$, respectively.

\section{Computing the hyper-Wiener index of zigzag polyhex nanotubes}

In this section we derive an exact formula for the hyper-Wiener index of $G:=T U H_{6}[2 p, q]$. For $u \in V(G)$ we define

$d(u)=\sum_{v \in V(G)} d(u, v), \quad d^{\prime}(u)=\sum_{v \in V(G)} d(u, v)^{2}, \quad d d(u)=\sum_{u \in V(G)}\left[d(u)+d^{\prime}(u)\right]$.

(If necessary, we show these quantities by $d_{G}(u), d_{G}^{\prime}(u)$ and $d d_{G}(u)$, respectively.) Then

$$
W W(G)=\frac{1}{4} \sum_{u \in V(G)} d(u)+\frac{1}{4} \sum_{u \in V(G)} d^{\prime}(u)=\frac{1}{4} \sum_{u \in V(G)} d d(u) .
$$

Also, for $u, v \in V(G)$, we define the hyper distance $d d(u, v)\left(\right.$ or $\left.d d_{G}(u, v)\right)$ as

$$
d d(u, v)=d(u, v)+d(u, v)^{2} .
$$

In the following lemma we find a formula for the hyper distances of one white (black) vertex of level zero of the graph $T U H_{6}[2 p, q]$ to all vertices on the level $k<q$ (see Figure 1). 
LEMMA 2.1. In the graph $T U H_{6}[2 p, q]$ we have

$$
\begin{aligned}
w w_{k} & :=\sum_{x \in \text { level } k} d d\left(x_{02}, x\right) \\
& =\sum_{x \in \text { level } k} d d\left(x_{04}, x\right) \\
& \vdots \\
& = \begin{cases}\frac{10}{3} k^{3}+\frac{2}{3} p^{3}+5 k^{2}+2 k^{2} p+2 k p+p^{2}+2 k p^{2}+\frac{5}{3} k+\frac{1}{3} p & \text { if } 0 \leq k<p \\
2 p(2 k+1)^{2} & \text { if } p \leq k\end{cases}
\end{aligned}
$$

and

$$
\begin{aligned}
b b_{k} & :=\sum_{x \in \text { level } k} d d\left(x_{01}, x\right) \\
& =\sum_{x \in \text { level } k} d d\left(x_{03}, x\right) \\
& \vdots \\
& = \begin{cases}\frac{10}{3} k^{3}+\frac{2}{3} p^{3}-3 k^{2}+2 k^{2} p+2 k p+p^{2}+2 k p^{2}-\frac{1}{3} k+\frac{1}{3} p & \text { if } 0 \leq k<p \\
8 k^{2} p & \text { if } p \leq k .\end{cases}
\end{aligned}
$$

PROOF. We compute $b_{k}$. It is sufficient to consider $x_{01}$. For other black vertices, the argument is similar. At first note that the lattice is symmetric (with respect to the line joining $x_{01}$ to $x_{11}$ ). We distinguish between three cases.

Case 1: $k \geq p$ and $k$ is even. In this case, for all $1 \leq j \leq p+1$,

$$
d\left(x_{01}, x_{k j}\right)= \begin{cases}2 k-1 & \text { if } j \text { is even } \\ 2 k & \text { if } j \text { is odd }\end{cases}
$$

By considering these vertices and their symmetric vertices we obtain $p$ vertices having distance $2 k-1$ from $x_{01}$, and $p$ vertices having $2 k$ distance from $x_{01}$. So

$$
\begin{aligned}
\sum_{u \in \text { level } k} & {\left[d\left(x_{01}, u\right)+d\left(x_{01}, u\right)^{2}\right] } \\
= & \sum_{j \text { is even }}\left[d\left(x_{01}, x_{j i}\right)+d\left(x_{01}, x_{j i}\right)^{2}\right]+\sum_{j \text { is odd }}\left[d\left(x_{01}, x_{j i}\right)+d\left(x_{01}, x_{j i}\right)^{2}\right] \\
\quad= & p\left[(2 k-1)+(2 k-1)^{2}\right]+p\left[(2 k)+(2 k)^{2}\right]=8 k^{2} p .
\end{aligned}
$$

Case 2: $k \geq p$ and $k$ is odd. In this case, for all $1 \leq j \leq p+1$,

$$
d\left(x_{01}, x_{k j}\right)= \begin{cases}2 k & \text { if } j \text { is even } \\ 2 k-1 & \text { if } j \text { is odd. }\end{cases}
$$


Now by considering these vertices and their symmetric vertices we obtain $p$ vertices having distance $2 k-1$ from $x_{01}$, and $p$ vertices having $2 k$ distance from $x_{01}$. So

$$
\begin{aligned}
\sum_{u \in \text { level } k} & {\left[d\left(x_{01}, u\right)+d\left(x_{01}, u\right)^{2}\right] } \\
= & \sum_{j \text { is even }}\left[d\left(x_{01}, x_{j i}\right)+d\left(x_{01}, x_{j i}\right)^{2}\right]+\sum_{j \text { is odd }}\left[d\left(x_{01}, x_{j i}\right)+d\left(x_{01}, x_{j i}\right)^{2}\right] \\
= & \left.p\left[(2 k)+(2 k)^{2}\right]+p[(2 k-1)]+(2 k-1)^{2}\right]=8 k^{2} p .
\end{aligned}
$$

Case 3: $k \leq p-1$. For all $p+1 \leq j$ and $j>k+1$,

$$
d\left(x_{01}, x_{k j}\right)=k+j-1 .
$$

Thus, the summation of the distances between $x_{01}$ and $x_{k j}$ (for all $j$ such that $p+1 \leq j$ and $j>k+1)$ and their symmetric vertices is

$$
\begin{aligned}
S_{1} & =2 \sum_{j=k+2}^{p}\left[(k+j-1)+(k+j-1)^{2}\right]+\left[(k+p+1-1)+(k+p+1-1)^{2}\right] \\
& =\frac{2}{3}(p-1-k)\left(p^{2}+4 p k+p+7 k^{2}+5 k\right)+(k+p)+(k+p)^{2} \\
& =2 k p+\frac{1}{3} p+2 p k^{2}+2 p^{2} k-7 k^{2}-\frac{7}{3} k+p^{2}-\frac{14}{3} k^{3}+\frac{2}{3} p^{3} .
\end{aligned}
$$

Also, if $1 \leq j \leq k+1$, then

$$
d\left(x_{01}, x_{k j}\right)= \begin{cases}2 k & \text { if } k-j \text { is odd } \\ 2 k-1 & \text { if } k-j \text { is even }\end{cases}
$$

Arguing as before we obtain $k+1$ vertices having distance $2 k$ and $k$ vertices having distance $2 k-1$ from $x_{01}$, respectively. Therefore, the summation of the distances between $x_{01}$ and $x_{k j}$ (for all $j$ such that $1 \leq j \leq k+1$ ) and their symmetric vertices is

$$
S_{2}=(k+1)\left[(2 k)+(2 k)^{2}\right]+k\left[(2 k-1)+(2 k-1)^{2}\right] .
$$

Hence,

$$
\begin{aligned}
b b_{k}= & S_{1}+S_{2} \\
= & 2 k p+\frac{1}{3} p+2 p k^{2}+2 p^{2} k-7 k^{2}-\frac{7}{3} k+p^{2}-\frac{14}{3}+\frac{2}{3} p^{3} \\
& +(k+1)\left[(2 k)+(2 k)^{2}\right]+k\left[(2 k-1)+(2 k-1)^{2}\right] \\
= & \frac{10}{3} k^{3}+\frac{2}{3} p^{3}-3 k^{2}+2 k^{2} p+2 k p+p^{2}+2 k p^{2}-\frac{1}{3} k+\frac{1}{3} p .
\end{aligned}
$$

In a similar manner we can compute $\widehat{w}_{k}$. 
COROllary 2.2. We have:

(a) $d d\left(x_{02}\right)=d d\left(x_{04}\right)=\cdots=d d\left(x_{0,2 p}\right)=w w_{0}+w w_{1}+\cdots+w w_{q-1}$;

(b) $d d\left(x_{01}\right)=d d\left(x_{03}\right)=\cdots=d d\left(x_{0,2 p-1}\right)=b b_{0}+b b_{1}+\cdots+b b_{q-1}$.

PROOF. By Lemma 2.1 we have

$$
\begin{aligned}
d d\left(x_{02}\right) & =\sum_{u \in \text { level } 0} d d\left(u, x_{02}\right)+\sum_{u \in \text { level } 1} d d\left(u, x_{02}\right)+\cdots+\sum_{u \in \text { level } q-1} d d\left(u, x_{02}\right) \\
& =w w_{0}+w w_{1}+\cdots+w w_{q-1}
\end{aligned}
$$

and so $\widehat{d}\left(x_{01}\right)=\widehat{d}\left(x_{03}\right)=\cdots=\widehat{d}\left(x_{0,2 p 2}\right)=\widehat{b}_{0}+\widehat{b}_{1}+\cdots+\widehat{b}_{q-1}$. The proof of (b) is similar.

LEMMA 2.3. If $0 \leq j \leq q-1$ is an odd number, then

(a)

$$
\begin{aligned}
d d_{G}\left(x_{j 1}\right) & =d d_{G}\left(x_{j 3}\right)=\cdots=d d_{G}\left(x_{j, 2 p-1}\right) \\
& =w w_{0}+w w_{1}+\cdots+w w_{q-(j+1)}+b b_{1}+\cdots+b b_{j}
\end{aligned}
$$

(b) $\quad d d_{G}\left(x_{j 2}\right)=d d_{G}\left(x_{j 4}\right)=\cdots=d d_{G}\left(x_{j, 2 p}\right)$

$$
=b b_{0}+b b_{1}+\cdots+b b_{q-(j+1)}+w w_{1}+\cdots+w w_{j} .
$$

PROOF. First suppose that $j=1$. We consider the tube that can be built up from two halves collapsing at level one. The bottom part is the graph $G_{1}=T U H C_{6}[2 p, q-1]$ and we can consider $x_{11}$ as one of the white edges in the first row of the graph $G_{1}$. According to Corollary 2.2,

$$
d d_{G_{1}}\left(x_{11}\right)=d d_{G_{1}}\left(x_{13}\right)=\cdots=d d_{G_{1}}\left(x_{1,2 p-1}\right)=w w_{0}+w w_{1}+\cdots+w w_{q-2} .
$$

The top part is the graph $T U H_{6}[2 p, 2]=\widehat{G_{1}}$, level one of graph $G$ is the first its row and $x_{11}$ is, as such, a black vertex of $\widehat{G_{1}}$. Therefore, by Corollary 2.2, $d d_{\widehat{G}_{1}}\left(x_{11}\right)=b b_{0}+b b_{1}$ and

$$
d d_{\widehat{G_{1}}}\left(x_{11}\right)=d d_{\widehat{G_{1}}}\left(x_{13}\right)=\cdots=d d_{\widehat{G_{1}}}\left(x_{1(2 p-1)}\right)=b b_{0}+b b_{1} .
$$

Since $w w_{0}=b b_{0}$ and $d d_{G}\left(x_{11}\right)=d d_{G_{1}}\left(x_{11}\right)+d d_{\widehat{G}_{1}}\left(x_{11}\right)-b b_{0}$, we have $d d_{G}\left(x_{11}\right)$ $=w w_{0}+\cdots+w w_{q-2}+b b_{1}$ and, similarly,

$$
d d_{G}\left(x_{11}\right)=d d_{G}\left(x_{13}\right)=\cdots=d d_{G}\left(x_{1,2 p-1}\right)=w w_{0}+\cdots+w w_{q-2}+b b_{1} .
$$

Similarly, for $x_{12}$ we can see that

$$
d d_{G}\left(x_{12}\right)=d d_{G}\left(x_{14}\right)=\cdots=d d_{G}\left(x_{1,2 p}\right)=b b_{0}+\cdots+b b_{q-2}+w w_{1} .
$$

By repetition of this argument, we obtain the result. 
LEMMA 2.4. If $0 \leq j \leq q-1$ is an even number, then

(a)

$$
\begin{aligned}
d d_{G}\left(x_{j 1}\right) & =d d_{G}\left(x_{j 3}\right)=\cdots=d d_{G}\left(x_{j, 2 p-1}\right) \\
& =b b_{0}+b b_{1}+\cdots+b b_{q-(j+1)}+w w_{1}+\cdots+w w_{j}
\end{aligned}
$$

(b) $\quad d d_{G}\left(x_{j 2}\right)=d d_{G}\left(x_{j 4}\right)=\cdots=d d_{G}\left(x_{j, 2 p}\right)$

$$
=w w_{0}+w w_{1}+\cdots+w w_{q-(j+1)}+b b_{1}+\cdots+b b_{j} .
$$

PROOF. First suppose that $j=2$. We consider the tube can be built up from two halves collapsing at level two. The bottom part is the graph $G_{2}=T U H_{6}[2 p, q-2]$, level two of $G$ is the first level of $G_{2}$ and we can consider $x_{21}$ as one of the black edges in the first row of graph $G_{2}$. According to Corollary 2.2,

$$
d d_{G_{2}}\left(x_{21}\right)=d d_{G_{2}}\left(x_{23}\right)=\cdots=d d_{G_{2}}\left(x_{2,2 p-1}\right)=b b_{0}+b b_{1}+\cdots+b b_{q-3} .
$$

The top part is the graph $T U H_{6}[2 p, 3]=\widehat{G_{2}}$, level two of graph $G$ is the first level of $\widehat{G_{2}}$ and $x_{21}$ is, as such, a white vertex of $\widehat{G_{2}}$. Therefore, by Corollary 2.2,

$$
d d_{\widehat{G_{2}}}\left(x_{21}\right)=w w_{0}+w w_{1}+w w_{2}
$$

and

$$
d d_{\widehat{G_{2}}}\left(x_{21}\right)=d d_{\widehat{G_{2}}}\left(x_{23}\right)=\cdots=d d_{\widehat{G_{2}}}\left(x_{2,2 p-1}\right)=w w_{0}+w w_{1}+w w_{2} .
$$

Since $w w_{0}=b b_{0}$ and $d d_{G}\left(x_{21}\right)=d d_{G_{2}}\left(x_{21}\right)+d d_{G_{2}}\left(x_{21}\right)-w w_{0}$, then

$$
d d_{G}\left(x_{21}\right)=b b_{0}+\cdots+b b_{q-3}+w w_{1}+w w_{2}
$$

and, similarly,

$$
d d_{G}\left(x_{21}\right)=d d_{G}\left(x_{23}\right)=\cdots=d d_{G}\left(x_{2,2 p-1}\right)=b b_{0}+\cdots+b b_{q-3}+w w_{1}+w w_{2} .
$$

We can repeat the process in a similar way for $x_{22}$ and see that

$$
d d_{G}\left(x_{22}\right)=d d_{G}\left(x_{24}\right)=\cdots=d d_{G}\left(x_{2,2 p}\right)=w w_{0}+\cdots+w w_{q-3}+b b_{1}+b b_{2} .
$$

By repetition of this argument we obtain the result.

For all $0 \leq j \leq q-1$, put

$$
\begin{gathered}
f(j)=w w_{0}+w w_{1}+\cdots+w w_{q-(j+1)}+b b_{1}+\cdots+b_{j} \quad \text { and } \\
g(j)=b b_{0}+b b_{1}+\cdots+b b_{q-(j+1)}+w w_{1}+\cdots+w w_{j} .
\end{gathered}
$$

We are now in a position to prove the main result of the paper.

THEOREM 2.5. The hyper-Wiener index, $W W(G)$, of $G:=T U H C_{6}[2 p, q]$ nanotubes is given by

$$
\begin{aligned}
& \frac{p q}{12}\left(4 p^{2} q^{2}+4 p^{3} q-4 p^{2}+2 q^{4}-2 q^{2}+4 p q^{2}-4 p-q+6 p^{2} q+q^{3}+2 p q^{3}\right), \\
& \frac{p^{2}}{12}\left(-2 q^{2}-6 p q-p^{3}+p+8 q^{4}-2 p^{4}+6 p^{3} q+2 p^{2}+8 q^{3}-6 q+4 p^{2} q\right),
\end{aligned}
$$

respectively, according to whether $p \geq q$ or $p<q$. 
PRoOF. Let

$$
\begin{aligned}
A_{1}=\{i \mid 1 \leq i \leq 2 p, i \text { even }\}, & A_{2}=\{i \mid 1 \leq i \leq 2 p, i \text { odd }\}, \\
B_{1}=\{j \mid 0 \leq j \leq q-1, j \text { even }\}, & B_{2}=\{j \mid 0 \leq j \leq q-1, j \text { odd }\} .
\end{aligned}
$$

Then $W W(G)$ is equal to

$$
\begin{aligned}
\frac{1}{4} & \sum_{x_{j i} \in V(G)} d d\left(x_{j i}\right) \\
= & \frac{1}{4}\left[\sum_{j \in B_{1}} \sum_{i \in A_{1}} d d\left(x_{j i}\right)+\sum_{j \in B_{1}} \sum_{i \in A_{2}} d d\left(x_{j i}\right)+\sum_{j \in B_{2}} \sum_{i \in A_{1}} d d\left(x_{j i}\right)\right. \\
& \left.+\sum_{j \in B_{2}} \sum_{i \in A_{2}} d d\left(x_{j i}\right)\right] \\
= & \frac{1}{4}\left[\sum_{j \in B_{1}} \sum_{i \in A_{1}} f(j)+\sum_{j \in B_{1}} \sum_{i \in A_{2}} g(j)+\sum_{j \in B_{2}} \sum_{i \in A_{1}} g(j)+\sum_{j \in B_{2}} \sum_{i \in A_{2}} f(j)\right] \\
= & \frac{1}{4}\left[\sum_{j \in B_{1}} \sum_{i \in A_{1}} f(j)+\sum_{j \in B_{1}} \sum_{i \in A_{2}} g(j)+\sum_{j \in B_{2}} \sum_{i \in A_{1}} g(j)+\sum_{j \in B_{2}} \sum_{i \in A_{2}} f(j)\right] \\
= & \frac{1}{4}\left[\sum_{j \in B_{1}} f(j) \sum_{i \in A_{1}} 1+\sum_{j \in B_{1}} g(j) \sum_{i \in A_{2}} 1+\sum_{j \in B_{2}} g(j) \sum_{i \in A_{1}} 1\right. \\
& \left.+\sum_{j \in B_{2}} f(j) \sum_{i \in A_{2}} 1\right] \\
= & \frac{1}{4}\left[\sum_{j \in B_{1}} p f(j)+\sum_{j \in B_{1}} p g(j)+\sum_{j \in B_{2}} p g(j)+\sum_{j \in B_{2}} p f(j)\right] \\
= & \frac{p}{4}\left[\sum_{j \in B_{1}}(f(j)+g(j))+\sum_{j \in B_{2}}(f(j)+g(j))\right]=\frac{p}{4} \sum_{j=0}^{q-1}(f(j)+g(j)) .
\end{aligned}
$$

First we prove the formula for the case $p \geq q$. Then, for each $0 \leq k \leq q-1$,

$$
\begin{gathered}
w w_{k}=\frac{10}{3} k^{3}+\frac{2}{3} p^{3}+5 k^{2}+2 k^{2} p+2 k p+p^{2}+2 k p^{2}+\frac{5}{3} k+\frac{1}{3} p, \\
b b_{k}=\frac{10}{3} k^{3}+\frac{2}{3} p^{3}-3 k^{2}+2 k^{2} p+2 k p+p^{2}+2 k p^{2}-\frac{1}{3} k+\frac{1}{3} p .
\end{gathered}
$$

So

$$
\begin{aligned}
W W(G)= & \frac{p}{4} \sum_{j=0}^{q-1}(f(j)+g(j)) \\
= & \frac{p q}{12}\left(4 p^{2} q^{2}+4 p^{3} q-4 p^{2}+2 q^{4}-2 q^{2}+4 p q^{2}-4 p-q\right. \\
& \left.+6 p^{2} q+q^{3}+2 p q^{3}\right) .
\end{aligned}
$$


Now we consider the case $p<q$. We break down this case into three subcases: $2 p>q, 2 p<q$ and $2 p=q$. Let

$$
\begin{aligned}
& C_{1}:=\{0 \leq j \leq p-1 \mid 0 \leq q-j-1 \leq p-1\} \\
& C_{2}:=\{0 \leq j \leq p-1 \mid p \leq q-j-1 \leq q-1\} \\
& C_{3}:=\{p \leq j \leq q-1 \mid 0 \leq q-j-1 \leq p-1\} \\
& C_{4}:=\{p \leq j \leq q-1 \mid p \leq q-j-1 \leq q-1\} .
\end{aligned}
$$

We note that if $C_{1} \neq \emptyset$, then $2 p>q$. Also if $C_{4} \neq \emptyset$, then $2 p<q$. Therefore, first suppose that $C_{1} \neq \emptyset$. Then $C_{4}=\emptyset$ and $2 p>q$. Therefore, if $j \in C_{1}$, then

$$
\begin{aligned}
f(j)= & \sum_{k=0}^{q-j-1}\left(\frac{10}{3} k^{3}+\frac{2}{3} p^{3}+5 k^{2}+2 k^{2} p+2 k p+p^{2}+2 k p^{2}+\frac{5}{3} k+\frac{1}{3} p\right) \\
& +\sum_{k=1}^{j}\left(\frac{10}{3} k^{3}+\frac{2}{3} p^{3}-3 k^{2}+2 k^{2} p+2 k p+p^{2}+2 k p^{2}-\frac{1}{3} k+\frac{1}{3} p\right),
\end{aligned}
$$

if $j \in C_{2}$, then

$$
\begin{aligned}
f(j)= & \sum_{k=0}^{p-1}\left(\frac{10}{3} k^{3}+\frac{2}{3} p^{3}+5 k^{2}+2 k^{2} p+2 k p+p^{2}+2 k p^{2}+\frac{5}{3} k+\frac{1}{3} p\right) \\
& +\sum_{k=1}^{j}\left(\frac{10}{3} k^{3}+\frac{2}{3} p^{3}-3 k^{2}+2 k^{2} p+2 k p+p^{2}+2 k p^{2}-\frac{1}{3} k+\frac{1}{3} p\right) \\
& +\sum_{k=p}^{q-j-1} 2 p(2 k+1)^{2},
\end{aligned}
$$

and if $j \in C_{3}$, then

$$
\begin{aligned}
f(j)= & \sum_{k=0}^{q-j-1}\left(\frac{10}{3} k^{3}+\frac{2}{3} p^{3}+5 k^{2}+2 k^{2} p+2 k p+p^{2}+2 k p^{2}+\frac{5}{3} k+\frac{1}{3} p\right) \\
& +\sum_{k=1}^{p-1}\left(\frac{10}{3} k^{3}+\frac{2}{3} p^{3}-3 k^{2}+2 k^{2} p+2 k p+p^{2}+2 k p^{2}-\frac{1}{3} k+\frac{1}{3} p\right) \\
& +\sum_{k=p}^{j} 8 k^{2} p .
\end{aligned}
$$

Also if $j \in C_{1}$, then

$$
\begin{aligned}
g(j)= & \sum_{k=0}^{j-1}\left(\frac{10}{3} k^{3}+\frac{2}{3} p^{3}+5 k^{2}+2 k^{2} p+2 k p+p^{2}+2 k p^{2}+\frac{5}{3} k+\frac{1}{3} p\right) \\
& +\sum_{k=1}^{q-j-1}\left(\frac{10}{3} k^{3}+\frac{2}{3} p^{3}-3 k^{2}+2 k^{2} p+2 k p+p^{2}+2 k p^{2}-\frac{1}{3} k+\frac{1}{3} p\right),
\end{aligned}
$$


if $j \in C_{2}$, then

$$
\begin{aligned}
g(j)= & \sum_{k=0}^{j}\left(\frac{10}{3} k^{3}+\frac{2}{3} p^{3}+5 k^{2}+2 k^{2} p+2 k p+p^{2}+2 k p^{2}+\frac{5}{3} k+\frac{1}{3} p\right) \\
& +\sum_{k=1}^{p-1}\left(\frac{10}{3} k^{3}+\frac{2}{3} p^{3}-3 k^{2}+2 k^{2} p+2 k p+p^{2}+2 k p^{2}-\frac{1}{3} k+\frac{1}{3} p\right) \\
& +\sum_{k=p}^{q-j-1} 8 k^{2} p,
\end{aligned}
$$

and if $j \in C_{3}$, then

$$
\begin{aligned}
g(j)= & \sum_{k=0}^{p}\left(\frac{10}{3} k^{3}+\frac{2}{3} p^{3}+5 k^{2}+2 k^{2} p+2 k p+p^{2}+2 k p^{2}+\frac{5}{3} k+\frac{1}{3} p\right) \\
& +\sum_{k=1}^{q-j-1}\left(\frac{10}{3} k^{3}+\frac{2}{3} p^{3}-3 k^{2}+2 k^{2} p+2 k p+p^{2}+2 k p^{2}-\frac{1}{3} k+\frac{1}{3} p\right) \\
& +\sum_{k=p}^{j} 2 p(2 k+1)^{2} .
\end{aligned}
$$

Therefore,

$$
\begin{aligned}
W W(G)= & \frac{p}{4} \sum_{j=0}^{q-1}[f(j)+g(j)] \\
= & \frac{p}{4}\left(\sum_{j \in C_{1}}[f(j)+g(j)]+\sum_{j \in C_{2}}[f(j)+g(j)]+\sum_{j \in C_{3}}[f(j)+g(j)]\right) \\
= & \frac{p^{2}}{12}\left(-2 q^{2}-6 p q-p^{3}+p+8 q^{4}-2 p^{4}+6 p^{3} q+2 p^{2}+8 q^{3}-6 q\right. \\
& \left.+4 p^{2} q\right),
\end{aligned}
$$

and so the formula is true for the case $p<q$ and $2 p>q$. Using this method we can handle other conditions.

\section{Acknowledgement}

The second author was partially supported by Center of Excellence of Mathematics of Isfahan University of Technology (CEAMA).

\section{References}

[1] A. R. Ashrafi and A. Loghman, "PI index of zig-zag polyhex nanotubes", MATCH Commun. Math. Comput. Chem. 55 (2006) 447-452.

[2] A. R. Ashrafi and A. Loghman, "PI index of armchair polyhex nanotubes", Ars Combin. 80 (2006) 193-199. 
[3] A. R. Ashrafi and S. Yousefi, "Computing the Wiener index of a $T U C_{4} C_{8}(\mathrm{~S})$ nanotorus", $M A T C H$ Commun. Math. Comput. Chem. 57 (2007) 403-410.

[4] J. Devillers and A. Balaban, Topological indices and related descriptors in QSAR and QSPR (Gordon and Breach, Amsterdam, 1999).

[5] M. V. Diudea, "Walk numbers ${ }^{e} W_{M}$ : Wiener numbers of higher rank", J. Chem. Inf. Comput. Sci. 36 (1996) 535-540.

[6] M. V. Diudea, "Wiener and hyper-Wiener numbers in a single matrix", J. Chem. Inf. Comput. Sci. 36 (1996) 833-836.

[7] M. V. Diudea, "Graphenes from 4-valent tori”, Bull. Chem. Soc. Jpn. 75 (2002) 487-492.

[8] M. V. Diudea, "Hosoya polynomial in tori", MATCH Commun. Math. Comput. Chem. 45 (2002) 109-122.

[9] M. V. Diudea and A. Graovac, "Generation and graph-theoretical properties of $C_{4}$-tori", $M A T C H$ Commun. Math. Comput. Chem. 44 (2001) 93-102.

[10] M. V. Diudea and P. E. John, "Covering polyhedral tori", MATCH Commun. Math. Comput. Chem. 44 (2001) 103-116.

[11] M. V. Diudea, I. Silaghi-Dumitrescu and B. Parv, "Toranes versus Torenes", MATCH Commun. Math. Comput. Chem. 44 (2001) 117-133.

[12] M. V. Diudea, M. Stefu, B. Parv and P. E. John, "Wiener index of armchair polyhex nanotubes", Croat. Chem. Acta 77 (2004) 111-115.

[13] A. A. Dobrynin, R. Entringer and I. Gutman, "Wiener index of trees: theory and applications", Acta Appl. Math. 66 (2001) 211-249.

[14] A. A. Dobrynin, I. Gutman, S. Klavžar and P. Žigert, "Wiener index of hexagonal systems", Acta Appl. Math. 72 (2002) 247-294.

[15] E. Eliasi and B. Taeri, "Szeged and Balaban indices of zig-zag polyhex nanutubes", MATCH Commun. Math. Comput. Chem. 56 (2006) 383-402.

[16] E. Eliasi and B. Taeri, "Balaban index of zigzag polyhex nanotorus", J. Comput. Theor. Nano Sci. 4 (2007) 1174-1178.

[17] E. Eliasi and B. Taeri, "Hyper-Wiener index of zigzag polyhex nanotorus", Ars Combin. 85 (2007) 307-318.

[18] F. Harary, Graph Theory (Addison-Wesley, Reading, MA, 1972).

[19] A. Heydari and B. Taeri, "Szeged Index of $T U C_{4} C_{8}(R)$ nanotubes", MATCH Commun. Math. Comput. Chem. 57 (2007) 463-477.

[20] A. Heydari and B. Taeri, "Wiener and Schultz Indices of $T U C_{4} C_{8}(R)$ nanotubes", J. Comput. Theor. Nano Sci. 4 (2007) 158-167.

[21] A. Heydari and B. Taeri, "Wiener and Schultz Indices of $T U C_{4} C_{8}(S)$ nanotubes", MATCH Commun. Math. Comput. Chem. 57 (2007) 665-676.

[22] A. Heydari and B. Taeri, "Hyper-Wiener index of $T U C_{4} C_{8}(S)$ nanotubes", J. Comp. Thoer. NanoSci. 5 (2008) 2275-2279.

[23] P. E. John and M. V. Diudea, "Wiener index of zig-zag polyhex nanotubes", Croat. Chem. Acta 77 (2004) 127-132.

[24] S. Klavžar, P. Žigert and I. Gutman, "An algorithm for the calculation of the hyper-Wiener index of benzenoid hydrocarbons", MATCH Commun. Math. Comput. Chem. 24 (2000) 229-233.

[25] D. J. Klein, I. Lukovits and I. Gutman, "On the definition of the hyper-Wiener index for cyclecontaining structures”, J. Chem. Inf. Comput. Sci. 35 (1995) 50-52.

[26] M. Randić, "Novel molecular descriptor for structure-property studies", Chem. Phys. Lett. 211 (1993) 478-483.

[27] H. Wiener, "Structural determination of paraffin boiling points", J. Am. Chem. Soc. 69 (1947) $17-20$.

[28] H. Wiener, "Correlation of heats of isomerization and differences in heats of vaporization of isomers among the paraffin hydrocarbons", J. Am. Chem. Soc. 69 (1947) 2636-2638.

[29] H. Wiener, "Influence of interatomic forces on paraffin properties", J. Chem. Phys. 15 (1947) 766-766. 
[30] H. Wiener, "Vapor pressure-temperature relationships among the branched paraffin hydrocarbons", J. Phys. Chem. 52 (1948) 425-430.

[31] H. Wiener, "Relation of the physical properties of the isomeric alkanes to molecular structure", J. Phys. Chem. 52 (1948) 1082-1089.

[32] S. Wolfram, The Mathematica $®$ book, 5th edn (Wolfram Media, Champaign, IL, 2003).

[33] S. Yousefi and A. R. Ashrafi, "An exact expression for the Wiener index of a polyhex nanotorus", MATCH Commun. Math. Comput. Chem. 56 (2006) 169-178.

[34] S. Yousefi and A. R. Ashrafi, "An exact expression for the Wiener index of a $T U C_{4} C_{8}(\mathrm{R})$ nanotorus", J. Math. Chem. 42 (2007) 1031-1039. 